\title{
NON-TRAUMATIC MYCOTIC KERATITIS
}

\author{
LAWRENCE W. HIRST, ${ }^{1}$ ALLAN SEBBAN, ${ }^{1}$ R. MICHAEL WHITBY, ${ }^{2}$ GRAEME R. NIMMO ${ }^{3}$ and \\ KEN STALLARD ${ }^{3}$ \\ Queensland, Australia
}

\begin{abstract}
SUMMARY
Two patients presented with culture proven Paecilomyces corneal infection, and a further patient with histologic evidence of fungal infection, on deep corneal biopsy. In all three cases the corneal infection was macroscopically present only in the depth of the cornea and on the endothelial surface with an intact epithelium and no overlying stromal involvement. Repeated surgery with large corneo-scleral grafts in two cases, and with medical therapy and a small patch-graft alone in the third case, resulted in long-term erradication of the infection and preservation of the globes. Antecedent modulation with steroid and/or cyclophosphamide may well have delayed the diagnosis, however, as there was no history of trauma in any of these cases, we postulate that these infections were not exogenously derived.
\end{abstract}

Microbial stromal keratitis without overlying epithelial defects have been reported as a result of infection by a number of organisms. ${ }^{1-4}$ Fungal keratitis without antecedent trauma has been reported rarely, ${ }^{1}$ while endogenous fungal endophthalmitis without previous trauma has been reported in immunocompromised patients. ${ }^{5}$ Paecilomyces has not been implicated in either of these types of infections and is best known for its role in exogenously induced endophthalmitis following the use of contaminated intraocular irrigating solutions. ${ }^{6.9}$ We report three cases of fungal keratitis not associated with trauma, presenting in an unusual fashion with the aetiologic agent in two of the cases being paecilomyces species and in the third being consistent with this organism.

From 'Division of Ophthalmology, Department of Surgery, The University of Queensland, 2nd Floor, Lions Building, Princess Alexandra Hospital, Ipswich Road, Woolloongabba, Queensland 4102, Australia; ${ }^{2}$ Director, Infectious Diseases, Princess Alexandra Hospital, Ipswich Road, Woolloongabba, Queensland 4102, Australia; ${ }^{3}$ Department of Microbiology, Princess Alexandra Hospital, Ipswich Road, Woolloongabba, Queensland 4102, Australia.

Correspondence to: Professor Lawrence W. Hirst, 2nd Floor, Lions Clinical Research Building, Princess Alexandra Hospital, Ipswich Road, Woolloongabba, Brisbane, Queensland 4102, Australia.

\section{CASE REPORTS}

\section{Case One}

A 21-year-old white male in excellent health and no history of intravenous drug abuse or intravenous therapy, had a two-month history of a sore right eye with no history of trauma or previous ocular disease. Six weeks after treatment with dexamethasone alcohol $0.1 \%$ drops, a small white patch developed on his cornea and he was referred for further consultation.

Examination revealed a grossly injected eye and a bilobed $3 \mathrm{~mm} \times 4 \mathrm{~mm}$ fluffy, white plaque which crossed the visual axis and was at the level of the endothelium and into the deep half of the stroma. There was one smaller lesion temporal to this main lesion which was strictly at the level of the endothelial surface and extending into the anterior chamber (Fig. 1). The overlying stroma was clear to high-power slit-lamp examination (Fig. 1) and the epithelium was intact over the entire cornea. His anterior chamber had many cells and considerable fibrin. His vision was hand motions only and the rest of the eye examination was normal.

Aqueous was aspirated and a deep corneal biopsy was taken. The cornea perforated during the operation and tissue adhesive was applied.

Histological examination of the deep biopsy revealed fungal elements and cultures grew Paecilomyces lilacinus from the cornea and anterior chamber fluid.

The patient was started on topical amphotericin B (5 micrograms $/ \mathrm{ml}$ ) hourly and intravenous amphotericin B was administered at a maintenance rate of $1.2 \mathrm{mg} / \mathrm{Kg} / 24$ hrs $(80 \mathrm{mg}$ daily) to a total dose of 2.0 grams. Ten days later the corneal infiltrate had increased dramatically involving almost the entire cornea (Fig. 2), and an $11 \mathrm{~mm}$ corneal transplant was performed removing all macroscopically involved corneal tissue. Post-operatively intravenous and topical amphotericin $B$ were continued and topical prednisolone acetate $1 \%$ every hour was started. After two weeks, the intravenous amphotericin B was discontinued and the eye remained quiet and the graft clear. Two weeks later, there was an apparent rejection of the

Eye (1992) 6, 391-395 


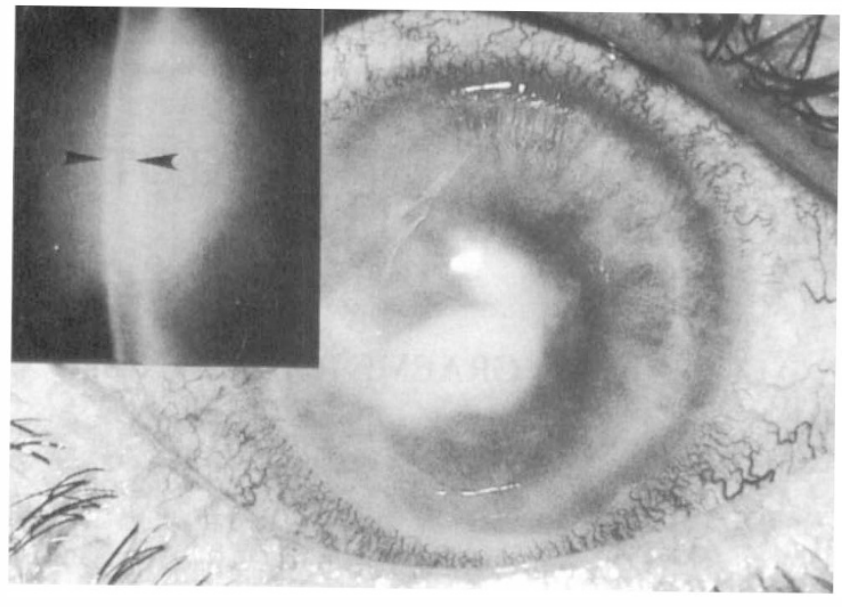

Fig. 1. Case One: External photograph of eye just prior to corneal biopsy revealing, deep stromal infiltrate and keratitic precipitates.

Inset: High magnification slit-lamp photograph revealing a clear corneal stroma overlying the deep infilitrate (between arrow heads).

graft and four weeks later, a $2 \mathrm{~mm}$ hypopyon appeared and there were fine "fluff" balls floating in the anterior chamber suggestive of renewed fungal infection (Fig. 3).

Aqueous grew Paecilomyces species and a two-week course of daily anterior chamber injections of 5 micrograms of amphotericin B was commenced. The graft became increasingly cloudy, but one month later an anterior chamber paracentesis revealed no growth.

Two weeks later, a presumptive diagnosis of recurrence of fungal infection was made after renewed anterior chamber activity and oral itraconazole was commenced at 400 $\mathrm{mg}$ daily and the topical amphotericin B was discontinued. A $12 \mathrm{~mm}$ corneo-scleral graft, resection of involved iris and an extracapsular cataract extraction were performed when the activity in cornea and anterior chamber increased. The iris and corneal specimens once again grew out Paecilomyces sp. but the lens did not. A further ten weeks of oral itraconazole, topical prednisone acetate

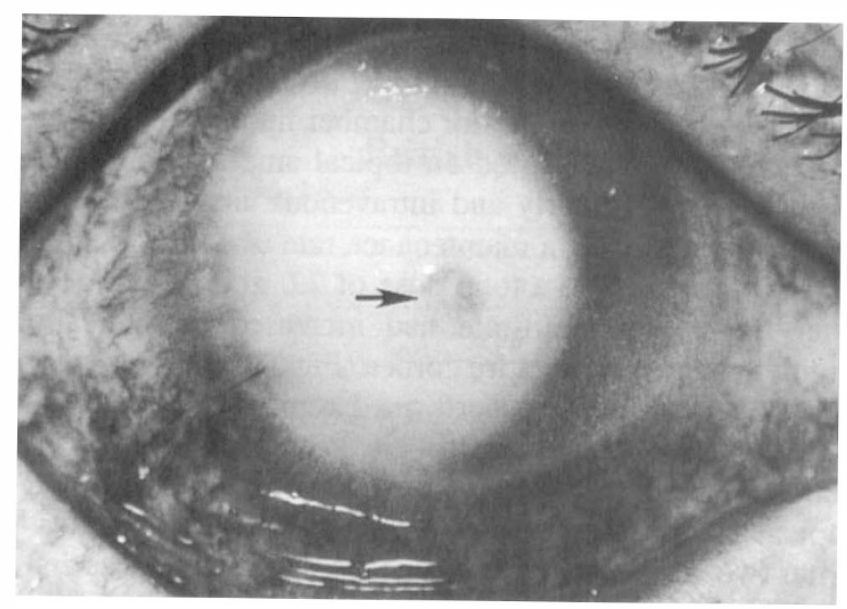

Fig. 2. Case One- External photograph of eye revealing extensive coalescent infiltrate and corneal biopsy site with tissue adhesive (arrow).
$1 \%$, topical gentamicin sulphate $0.3 \%$ and oral acetazolamide $250 \mathrm{mg}$ six hourly was continued.

Two years after the surgery, off all oral and topical medication, the eye has remained free from infection with an opaque graft, vision of $6 / 240$ and a pressure of $10 \mathrm{~mm}$ $\mathrm{Hg}$.

\section{Case Two}

A 72-year-old white female presented with a red, sore left eye with no previous history of trauma or intravenous therapy or drug abuse, and the only significant past medical history of rheumatoid arthritis. Examination revealed an elevated and injected nasal bulbar conjunctiva with a clear cornea and quiet anterior chamber. A diagnosis of episcleritis was made and she was started on topical prednisolone acetate $1 \%$ with an initial resolution of signs. However, one month later she returned with a more obvious nodule in her sclera which symptomatically improved on recommencing steroid therapy. Four weeks later her eye was comfortable, her vision was $6 / 6$ and the nodule was less marked.

Approximately two months later she returned with increasing pain, and there was now an area of deep corneal infiltrate in the limbal area adjacent to the area of previous scleral involvement. The overlying epithelium was intact and the superficial stroma over this infiltrate was clear by high-power slit-lamp examination. Her intraocular pressure was $35 \mathrm{mmHg}$. A diagnosis of rheumatoid sclerokeratitis was made and she was started on topical dexamethasone alcohol $0.1 \%$ every two hours, prednisolone acetate $1 \%$ six hourly, atropine sulphate $1 \%$ six hourly, timoptol maleate $0.5 \%$ twelve hourly and oral prednisone $50 \mathrm{mg}$ daily. After two weeks of therapy, she showed no response and was referred.

The eye had gross ciliary injection, there was generalized corneal oedema and a deep stromal infiltrate $2 \mathrm{~mm}$ in size superonasally extending from the endothelium into the anterior chamber and marked endothelial deposits in other areas (Fig. 4). There was an intact corneal epi-

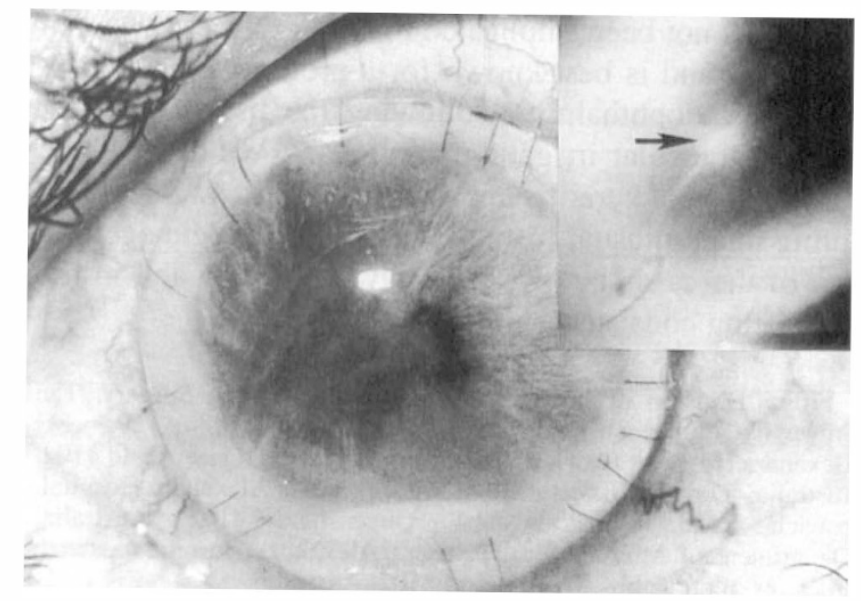

Fig. 3. Case One: External photograph revealing large corneoscleral graft, hypopyon and pupillary membrane. Inset: High magnification slit-lamp photograph of presumed fungal "fluff" ball (arrow). 


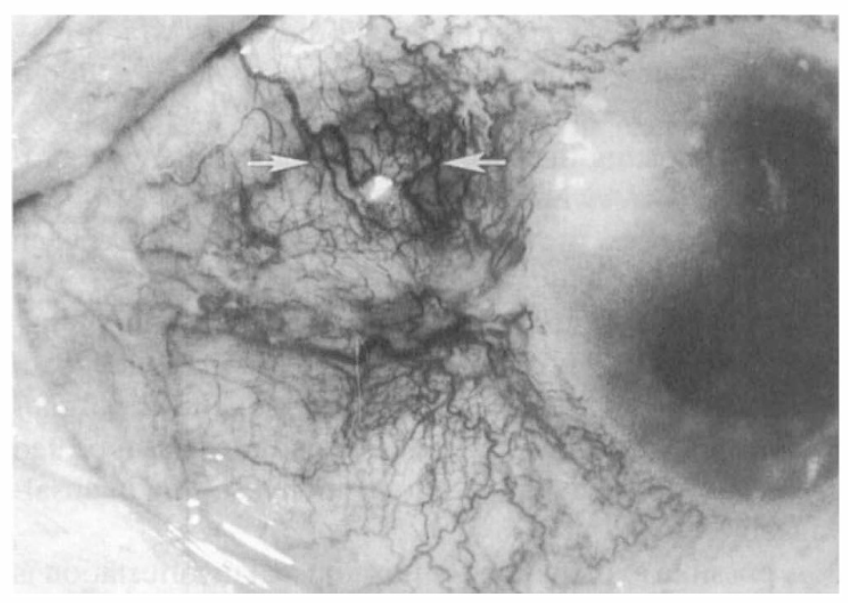

Fig. 4. Case Two: External photograph revealing elevated scleral mass (arrows) and adjacent corneal infiltrate.

thelium and the superficial stroma was clear. Nasally there was an area of thin sclera and the anterior chamber had considerable cellular activity and a large fibrin clot. Her intraocular pressure was $22 \mathrm{mmHg}$. The posterior segment appeared normal. The oral prednisone was increased to 80 $\mathrm{mg} /$ day and she was begun on cyclophosphamide $150 \mathrm{mg} /$ day. Within one week a large epithelial defect developed, and a deep corneal biopsy and anterior chamber paracentesis revealed septate fungal hyphae and spores. Cultures grew out Paecilomyces species.

Cyclophosphamide and prednisone were discontinued, topical prednisolone acetate $1 \%$ was continued, and topical amphotericin B (5 micrograms $/ \mathrm{ml}$ ) hourly together with intravenous amphotericin B administered at a maintenance rate of $0.8 \mathrm{mg} / \mathrm{Kg} / 24 \mathrm{hrs}$ ( $50 \mathrm{mg}$ daily) were commenced. Acetazolamide was started at $125 \mathrm{mg}$ eight hourly because the intraocular pressure rose to $50 \mathrm{mmHg}$. Within a week new stromal infiltrates had appeared and the patient underwent a $12 \mathrm{~mm}$ penetrating keratoplasty, a lens extraction and debridement of the anterior chamber and two peripheral iridectomies. The posterior capsule was left intact and the vitreous was noted to be clear. One week later there was an increase in what appeared to be a

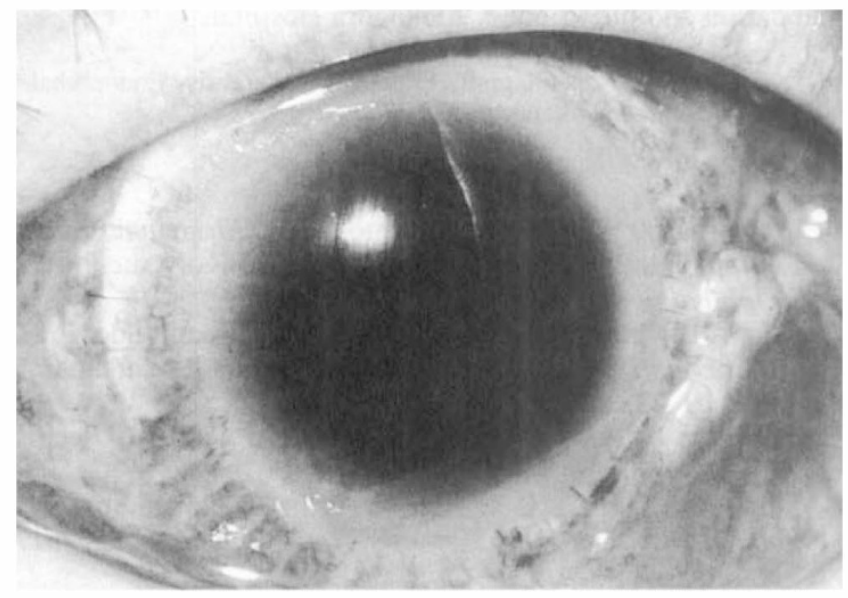

Fig. 5. Case Two: Large, well-healed oedematous corneoscleral graft with no residual iris tissue. mycelial mass filling the pupil, and a total pars plana vitrectomy, sub-total iridectomy, capsulotomy and a clearance of the anterior segment fungal mass was performed together with an irrigation of the intraocular cavity with amphotericin. For the next five days she received intravitreal injections of amphotericin B $0.2 \mathrm{ml}$ (5 micrograms/ $\mathrm{ml}$ ) and continued her intravenous amphotericin B therapy to a total dose of 0.7 grams. However, she tolerated the amphotericin therapy very poorly and this was discontinued. Three weeks after the intraocular clearance she had a large corneal epithelial defect for which a tarsorrhaphy was performed. Three months later, the graft was opaque but the eye was quiet (Fig. 5). Two years later, she has maintained light perception vision with a normal intraocular pressure and is off all medications.

\section{Case Three}

A 45-year-old white male presented with a history of increasing photophobia and epiphora for seven weeks in his right eye. He had no history of trauma or intravenous drug abuse, and had no previous ocular problems and was in excellent health. He was started on topical prednisolone acetate $1 \%$ and topical chloramphenicol $0.5 \%$ and was followed for five weeks with slight improvement of symptoms. However, he developed some deep corneal oedema and was referred for consultation.

On examination, his vision was $6 / 6$ in both eyes and his right eye had considerable ciliary injection, full thickness corneal oedema in a circular area near the inferotemporal limbus $3 \mathrm{~mm}$ in diameter with some keratitic precipitates on the posterior endothelial surface underlying the area of oedema. The corneal epithelium was intact. The rest of the stroma was clear. A diagnosis of right, herpetic disciform keratitis was made and the patient was started on an intensive course of topical prednisolone acetate $1 \%$ hourly and homatropine $2 \%$ six hourly. Five weeks later, his eye had deteriorated with an increased anterior chamber reaction, and two large mutton fat keratitic precipitates on the posterior endothelial surface. Five weeks later there was a new finding of a dense, localized corneal infiltrate in the area of previous oedema which extended from the pos-

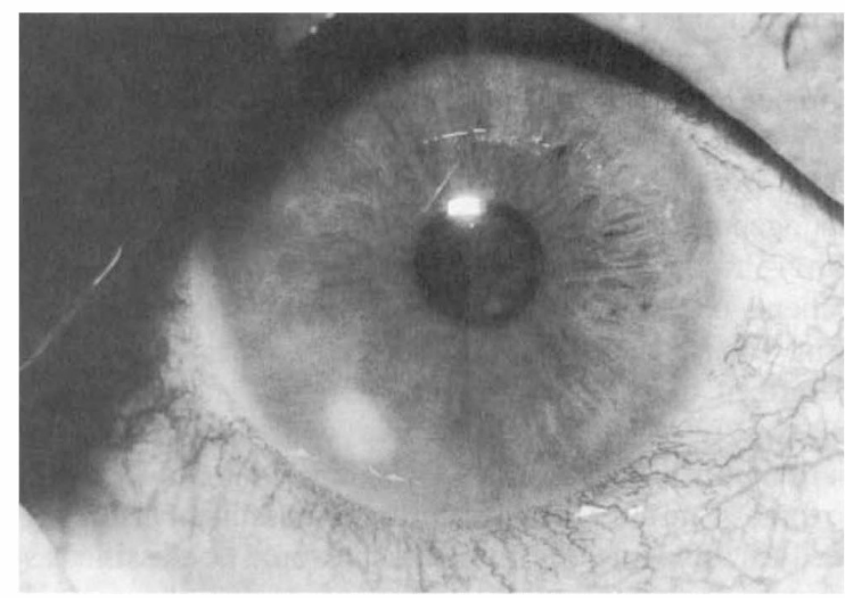

Fig. 6. Case Three: External photograph revealing localized deep stromal infiltrate. 
terior third of the cornea into the anterior chamber as an endothelial plaque (Fig. 6), with the superficial corneal stroma entirely clear and the epithelium intact. Gram stained sections of a deep corneal biopsy showed definite septate fungal hyphae but cultures were unsuccessful in isolating any organism from the cornea of aqueous.

Intravenous amphotericin B was started at a maintenance rate of $0.87 \mathrm{mg} / \mathrm{Kg} / 24 \mathrm{hrs}$ ( $60 \mathrm{mg}$ daily) but one week later the area of infiltrated stroma under the biopsy site perforated and the patient was given a full thickness cornel patch graft $2 \mathrm{~mm} \times 2 \mathrm{~mm}$ in size. After four further weeks of systemic amphotericin B (a total of 2.0 grams) the patient was discharged on topical amphotericin B 5 micrograms six hourly and atropine $1 \%$ eight hourly. Two years later, the patient is off all medications and the eye is quiet with a small, opaque patch graft and 6/6 vision and normal intraocular pressure.

\section{DISCUSSION}

There are a number of unique and interesting clinical observations with respect to these three cases of deep fungal keratitis. They all occurred in the absence of overt epithelial defects. This has been reported in one case of Fusarium solani keratitis and endophthalmitis ${ }^{1}$ and from a number of bacterial agents such as Neisseria species $^{2}$ and Corynebacterium species $^{3}$ and from stromal herpetic keratitis. $^{4}$

In addition, there was no obvious trauma to explain a deep inoculation of fungus into the cornea or anterior chamber. All three patients were examined early in the disease course and on slit-lamp examination showed no corneal infiltrates.

All specimens were inoculated onto six media (solid: blood incubated aerobically and anaerobically, MacConkey agar, Chocolate agar; liquid: thioglycollate and brain-heart-infusion broth; fungal: Saborouds dextrose agar incubated at $25^{\circ} \mathrm{C}$ and $47^{\circ} \mathrm{C}$ ). Forty-eight hours after inoculation, a filamentous fungus (later identified as Paecilomyces), was cultured on all liquid and fungal media as well as blood agar. This isolation pattern suggested the organism was a true pathogen and not a laboratory contaminant.

After varying intervals, all three cases developed deep corneal infiltrates in previously clear corneas. The overlying stroma in all three of these cases was remarkably clear by high-power slit-lamp examination. Although endogenous fungal endophthalmitis has been reported fairly frequently, principally in immunosuppressed patients or those on long-term intravenous therapy or in drug abusers ${ }^{5}$ endogenous keratitis has not been described. Two of our patients, cases one and three were healthy, relatively young people with no previous or current medical illnesses and who have now survived two to three years after the event with no subsequent history of medical problems. The third patient would be classified as immunocompromised by virtue of severe rheumatoid arthritis. On the other hand, all of the patients had been on immunosuppressive drugs including steroids and/or cyclophosphamide prior to fungal diagnosis which may well have altered the manifestation of a fungal endophthalmitis and keratitis.

It is interesting that two of the three patients were proven Paecilomyces infections.

Paecilomyces, previously known as Penicillium, is an ubiquitous saprophyte and has been reported as a contaminant of solutions causing exogenous endophthalmitis. ${ }^{6,7.9}$ Podedworny and Suie ${ }^{10}$ in 1964 were the first to report an ocular case of Paecilomyces, isolating the organism from a scleral lesion. It has been a rare but repeated cause of serious ocular infections often resulting in unsalvageable eyes $\mathrm{s}^{6.11 .12}$

A possible explanation for this unusual manifestation is that there has been inadvertent deep traumatic inoculation of the stroma, which has resulted in a slowly progressive, deep keratitis probably modified by the administration of immunosuppressive agents. However, it seems unlikely that such an injury would not be noticed by any of these patients.

There was remarkable persistence of infection in cases one and two where only extensive reconstructive surgery appears to have been able to remove the bulk of infection such that intravenous and topical antifungal agents were then effective. In case one, even after apparent erradication of the disease, a recurrence occurred three months later. In case two, erradication was finally achieved by extensive surgical intraocular debridement and intraocular irrigation with amphotericin B.

In cases one and two, massive anterior segment corneoscleral grafts had been instrumental in the surgical stabilization of these devastating infections. Although both of these grafts have become opaque the eyes have been stable for two years and are normo-tensive.

Further studies would be necessary to indicate why Paecilomyces infections, in particular, may manifest themselves in this unusual fashion.

The authors wish to thank Dr B. Wilson and Dr G. Hoole for referring patient one, Dr A. Lamb for patient two and Dr L. Piggot for patient three.

This work was supported in part by the Prevent Blindness Foundation and the Princess Alexandra Hospital.

Key words: Corneo-scleral graft, Endogenous keratitis, Endophthalmitis, Fungal, Infective keratitis, Paecilomyces.

\section{REFERENCES}

1. Lieberman TW, Ferry AP, Bottone EJ: Fusarium solani Endophthalmitis without primary corneal involvement. Am J Ophthalmol. 1979, 88: 764-7.

2. Robin JB: Gonococcal Ocular Disease. In: Fraunfelder F. T. ed, Current Ocular Therapy. W. B. Saunders, Philadelphia. 1990: 20-22.

3. Chandler JW and Milam DF: Diphtheria Corneal Ulcers. Arch Ophthalmol. 1978, 96: 53-6.

4. Stern GA, Zam ZS, Gutgesell VJ: Primary Herpes Simplex Subepithelial Dendritic Keratitis. Am J Ophthalmol. 1981. 91: 496-9.

5. Deutsch D, Adler S, Teller J, Savir H: Endogenous Candidal Endophthalmitis. Ann Ophthalmol. 1989, 21: 260-8.

6. Pettit TH, Olson RJ, Foos RY, Martin WJ: Fungal Endoph- 
thalmitis Following Intraocular Lens Implantation-A Surgical Epidemic. Arch Ophthalmol. 1980, 98: 1025-39.

7. O'Day DM: Fungal Endophthalmitis caused by Paecilomyces lilacinus After Intraocular Lens Implantation, Editorial. Am J Ophthalmol. 1977, 83: 130-1.

8. Miller GR, Rebell G, Magoon RC, Kulvin SM, Forster RK: Intravitreal Antimycotic Therapy and the Cure of Mycotic Endophthalmitis Caused by a Paecilomyces lilacinus Contaminated Pseudophakos. Ophthalmic Surgery. 1978, 9: 54-63.

9. Mosier MA, Lusk B, Pettit TH, Howard DH, Rhodes J: Fun- gal Endophthalmitis Following Intraocular Lens Implantation. Am J Ophthalmol. 1977, 83: 1-8.

10. Podedworny W, Suie T: Mycotic Infection of the Sclera. Am J Ophthalmol. 1964, 58: 494.

11. Rodrigues MM and MacLeod D: Exogenous Fungal Endophthalmitis Caused by Paecilomyces. Am J Ophthalmol. 1975, 79: 687-90.

12. Kozarsky AM, Stulting D, Waring GO, Cornell FM, Wilson LA, Cavanagh HD: Penetrating Keratoplasty for Exogenous Paecilomyces Keratitis Followed by Postoperative Endophthalmitis. Am J Ophthalmol. 1984, 98: 552-7. 